\title{
Obituary
}

\section{Prof. John Mallard, OBE, FRSE, FREng, FIPEM, FIUPESM}

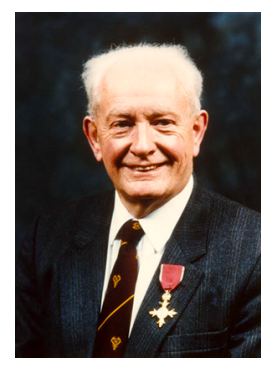

On 25 February 2021 the international medical physics community lost a very special member - Prof. John Mallard from Aberdeen, UK

Prof. John Mallard played a crucial role in the development of two of the world's most important medical technologies - Magnetic Resonance Imaging (MRI) and Positron Emission Tomography (PET). He also played important role in the international development of medical physics.

Prof. Mallard was born in 1927 in Northampton, UK. He obtained his $\mathrm{PhD}$ in the University College, Nottingham (at that time part of University of London). In 1959 he built the first whole-body isotope scanner in the UK. In 1964 Prof. Mallard published in the journal Nature his views related to medical use of MRI. In 1965 he was appointed at the University of Aberdeen. During 1970s Prof. Mallard built a team of specialists led by Dr Jim Hutchinson. This team developed an unique MRI scanner (0.04 Tesla) and applied it to imaging of laboratory animals. The team also built the first in the world full-body MRI scanner and on 26 April 1980 they scanned the first patient. In 1992 Prof. Mallard retired from the University of Aberdeen, UK.

Prof. John Mallard was very active in the international development of medical physics and biomedical engineering. He took part in the establishment of both - the International Organisation for Medical Physics (IOMP) and the International Union for Physical and Engineering Sciences in Medicine (IUPESM). From 1962 to 1965 he was the Founder Secretary General of IOMP, later from 1979-1982 he was IOMP President. In this period he was involved in the formation of the International Union for Physical and Engineering Sciences in Medicine (IUPESM) and in 1980 became its Founder President. In this position he made the first steps towards the recognition of the scientific fields medical physics and biomedical engineering by the International Council of Scientific Unions (ICSU - now ISC, The International Science Council).

Prof. John Mallard has received many awards. In 1992 he was appointed Officer of the Order of the British Empire in the Queen's Birthday Honours List. The UK IPEM established an annual lecture in his name; The American AAPM awarded him with the Landau Memorial Plaque; the IOMP included him in the list of 50 Outstanding medical physicists; the IUPESM awarded him their first Fellowship for International Leadership. In 2016 IOMP established an international Award The John Mallard Award which honours a medical physicist who has developed an innovation of high scientific quality and who has applied this innovation in clinical practice.

On behalf of the IOMP Executive Committee we are sending deepest condolences to Prof. John Mallard's family.

IOMP expresses heartfelt thanks to Prof. John Mallard for his enormous contribution to medical physics! 